\title{
Varenicline-induced Mixed Mood and Psychotic Episode in a Patient with Schizoaffective Disorder
}

\section{To the Editor:}

April 7, 2009

Varenicline is an $\alpha 4 \beta 2$ nicotinic cholinergic receptor ( $\mathrm{nAChR}$ ) partial agonist approved for smoking cessation. We present a patient with schizoaffective disorder, bipolar type, who received varenicline and experienced an activated manic episode and psychotic relapse.

A 43-year-old ethnic Chinese man suffering from schizoaffective disorder, bipolar type, had been treated for 20 years with clothiapine 160 $\mathrm{mg} /$ day and lithium 1,200 $\mathrm{mg} /$ day (lithium serum as $0.7 \mathrm{mmol} / \mathrm{L}$ ). He began smoking as a teenager and consumed one pack of cigarettes/day. A year ago, he was admitted to our psychiatric chronic ward for rehabilitation. Since our government recently implemented a "Tobacco Hazard Control Act", which forbids smoking in hospitals, the patient had to quit smoking and decided to use varenicline for smoking abstinence.

After 3 days of taking varenicline, he developed mild nausea. Ten days latter, a decreased need for sleep and elevated mood were noted. Persecutory delusions and auditory hallucination were also exacerbated. He was then transferred to psychiatric acute ward and varenicline was discontinued. His daily lithium dosage was increased to $1,500 \mathrm{mg}$ and blood serum level as $0.9 \mathrm{mmol} / \mathrm{L}$. He also took clonazepam $2 \mathrm{mg}$ four times a day, and clothiapine $160 \mathrm{mg} /$ day. After 4 weeks of treatment, his manic and psychotic symptoms resolved.

Varenicline was approved by the Food and Drug Administration in 2006 for smoking cessation; it was designed to selectively activate the $\alpha 4 \beta 2 \mathrm{nAChR}$, mimicking the action of nicotine and causing the release of dopamine. ${ }^{1}$ This should counteract the withdrawal symptoms consequent upon low dopamine release during smoking cessation. There have been case reports detailing exacerbation of schizophrenia by varenicline as well as a varenicline-induced manic episode in a patient with bipolar disorder. Agitation, euphoric mood, and exacerbation of psychotic symptoms were reported as infrequent psychiatric adverse reactions. ${ }^{2,3}$ To our knowledge, this case is the first report of mania with psychotic symptoms associated with varenicline use in schizoaffective disorder. Since varenicline might lead to the dysregulation of the dopaminergic system, varenicline might exacerbate psychotic symptoms in patients with psychotic disease..$^{2,3}$ In addition, varenicline also acts on neuronal $\alpha 7$ nAChR1, which has been linked to major psychiatric disorders.

Although our patient is an inpatient under lithium and clothiapine treatment, both manic episodes and psychotic symptoms developed after varenicline treatment, suggesting that lithium or antipsychotics may not prevent the psychotic relapse adverse effect with varenicline. The manic and psychotic symptoms improved with discontinuing varenicline and increasing lithium. With the high rate of smoking among psychiatric patients and the severe psychiatric adverse effects, ${ }^{2,3}$ the at-risk population that will probably be consumers of a medication should be included in the trials before that drug is approved.

Sincerely,

Mu-En Liu, MD, Shih-Jen Tsai, MD, and Szu-Tung Yang, MD

\section{REFERENCES}

1. Mihalak KB, Carroll FI, Luetje CW. Varenicline is a partial agonist at alpha4beta2 and a full agonist at alpha7 neuronal nicotinic receptors. Mol Pharmacol. 2006;70:801-805.

2. Freedman R. Exacerbation of schizophrenia by varenicline. Am J Psychiatry. 2007:164:1269.

3. Kohen I, Kremen N. Varenicline-induced manic episode in a patient with bipolar disorder. Am J Psychiatry. 2007;164:1269-1270.

Dr. Liv and Dr. Yang are both attending psychiatrists in the Department of Psychiatry at Longcyuan Veterans Hospital in Pingtung, Taiwan. Dr. Tsai is attending psychiatrist in the Department of Psychiatry at Taipei Veterans General Hospital and associate professor at the School of Medicine at National Yang-Ming University in Taiwan.

Disclosure: The authors do not have an affiliation with or financial interest in any organization that might pose a conflict of interest. 
Now, at the first sign of failure, count on VIMPAT-a first-in-class AED for the adjunctive treatment of partial-onset seizures.

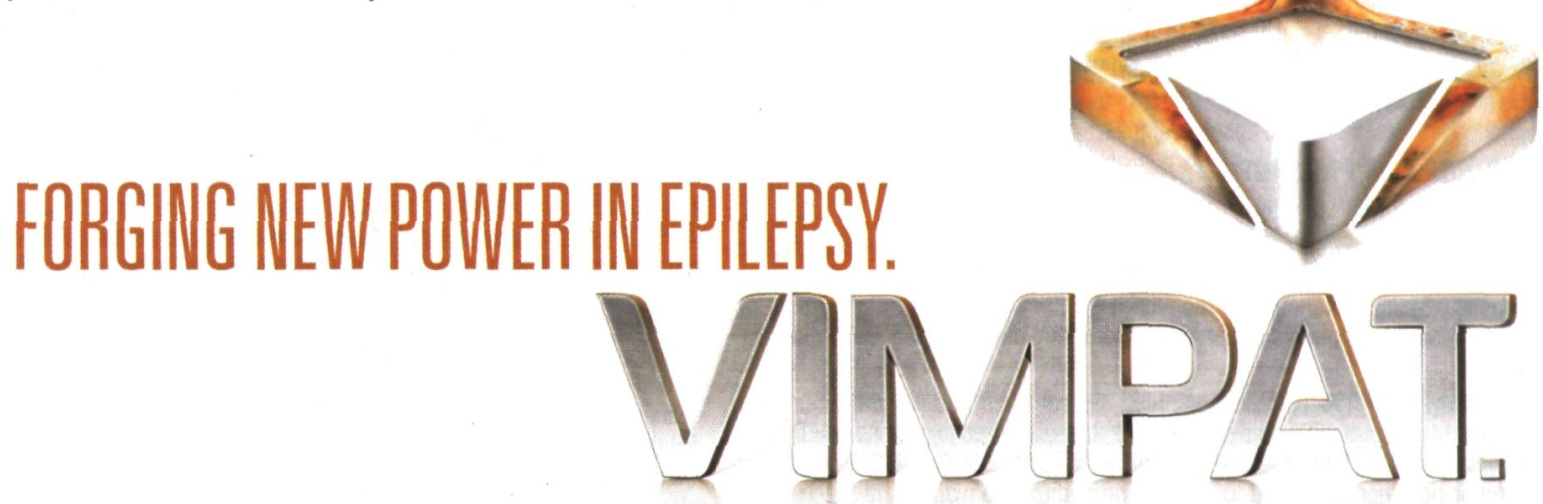

- Patients achieved greater reduction in seizure frequency

- Proven efficacy with the broadest range of AEDs*

- Power that was generally well tolerated

- Introduce a novel mechanism of action ${ }^{2}$

AVAILABLE IN ORAL TABLETS AND IV INJECTION.

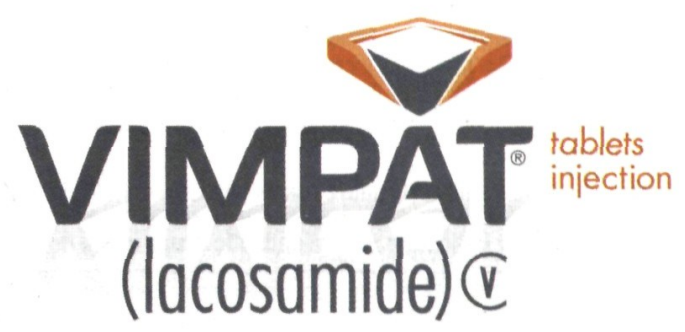

\section{IMPORTANT SAFETY INFORMATION}

VIMPAT ${ }^{\circledast}$ tablets are indicated as adjunctive therapy in the treatment of partial-onset seizures in patients with epilepsy who are 17 years and older. VIMPAT injection is indicated as short-term replacement when oral administration is temporarily not feasible in these patients. Patients should be advised that VIMPAT may cause dizziness, ataxia, and syncope. Caution is advised for patients with known cardiac conduction problems, who are taking drugs known to induce PR interval prolongation, or with severe cardiac disease. In patients with seizure disorders, VIMPAT should be gradually withdrawn to minimize the potential of increased seizure frequency. Multiorgan hypersensitivity reactions have been reported with antiepileptic drugs. If this reaction is suspected, treatment with VIMPAT should be discontinued.

AEDs increase the risk of suicidal behavior and ideation. Patients taking VIMPAT should be monitored for the emergence or worsening of depression, suicidal thoughts or behavior, and/or any unusual changes in mood or behavior.

The most common adverse reactions occurring in $\geq 10$ percent of VIMPAT-treated patients, and greater than placebo, were diplopia, headache, dizziness, and nausea.

Please see adjacent page for Brief Summary of full Prescribing Information.

*VIMPAT was tested with 2nd-generation AEDs including levetiracetam, lamotrigine, topiramate,

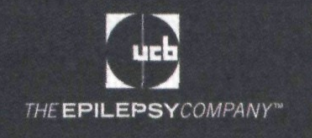

Farion. 2009, UCB, Inc., Smyrna, GA 30080 All rights reserved. Printed in U.S.A. VE150.1208 The Epilepsy Company is a trademark of the UCB Group of Companies. 


\section{VIMPAT ${ }^{\circledR}$ (lacosamide) Tablets, CV VIMPAT ${ }^{\oplus}$ (lacosamide) Injection, CV Brief Summary of Full Prescribing Information (See Package Insert for Full Prescribing Information) \\ Rx Only INDICATIONS AND USAGE \\ Partial-Onset Seizures}

VIMPAT (lacosamide) tablets are indicated as adjunctive therapy in the treatment of partial-onset seizures in patients with epilepsy aged 17 years and older.

VIMPAT (lacosamide) injection for intravenous use is indicated as adjunctive therapy in the treatment of partial-onset seizures in patients with epilepsy aged 17 years and older when oral administration is temporarily not feasible.

\section{CONTRAINDICATIONS}

None.

\section{WARNINGS AND PRECAUTIONS}

\section{Suicidal Behavior and Ideation}

Antiepileptic drugs (AEDs), including VIMPAT, increase the risk of suicidal thoughts or behavior in patients taking these drugs for any indication. Patients treated with any AED for any indication should be monitored for the emergence or worsening of depression, suicidal thoughts or behavior, and/or any unusual changes in mood or behavior.

Pooled analyses of 199 placebo-controlled clinical trials (mono- and adjunctive therapy) of 11 different AEDs showed that patients randomized to one of the AEDs had approximately twice the risk (adjusted Relative Risk 1.8, 95\% Cl:1.2, 2.7) of suicidal thinking or behavior compared to patients randomized to placebo. In these trials, which had a median treatment duration of 12 weeks, the estimated incidence of suicidal behavior or ideation among 27,863 AED-treated patients was $0.43 \%$, compared to $0.24 \%$ among 16,029 placebo-treated patients, representing an increase of approximately one case of suicidal thinking or behavior for every 530 patients treated. There were four suicides in drug-treated patients in the trials and none in placebo-treated patients, but the number of events is too small to allow any conclusion about drug effect on suicide.

The increased risk of suicidal thoughts or behavior with AEDs was observed as early as one week after starting treatment with AEDs and persisted for the duration of treatment assessed. Because most trials included in the analysis did not extend beyond 24 weeks, the risk of suicidal thoughts or behavior beyond 24 weeks could not be assessed.

The risk of suicidal thoughts or behavior was generally consistent among drugs in the data analyzed. The finding of increased risk with AEDs of varying mechanisms of action and across a range of indications suggests that the risk applies to all AEDs used for any indication. The risk did not vary substantially by age ( $5-100$ years) in the clinical trials analyzed.

Table 1 shows absolute and relative risk by indication for all evaluated AEDs.

Table 1 Risk by indication for antiepileptic drugs in the pooled analysis

\begin{tabular}{|l|l|l|l|l|}
\hline Indication & $\begin{array}{l}\text { Placebo } \\
\text { Patients with } \\
\text { Events Per } \\
1000 \text { Patients }\end{array}$ & $\begin{array}{l}\text { Drug Patients } \\
\text { with Events } \\
\text { Per 1000 } \\
\text { Patients }\end{array}$ & $\begin{array}{l}\text { Relative Risk: } \\
\text { Incidence of } \\
\text { Events in Drug } \\
\text { Patients/ } \\
\text { Incidence in } \\
\text { Placebo } \\
\text { Patients }\end{array}$ & $\begin{array}{l}\text { Risk } \\
\text { Difference: } \\
\text { Additional } \\
\text { Drug Patients } \\
\text { with Events } \\
\text { Per 1000 } \\
\text { Patients }\end{array}$ \\
\hline Epilepsy & 1.0 & 3.4 & 3.5 & 2.4 \\
\hline Psychiatric & 5.7 & 8.5 & 1.5 & 2.9 \\
\hline Other & 1.0 & 1.8 & 1.9 & 0.9 \\
\hline Total & 2.4 & 4.3 & 1.8 & 1.9 \\
\hline
\end{tabular}

The relative risk for suicidal thoughts or behavior was higher in clinical trials for epilepsy than in clinical trials for psychiatric or other conditions, but the absolute rișk differences were similar.

Anyone considering prescribing VIMPAT or any other AED must balance this risk with the risk of untreated illness. Epilepsy and many other illnesses for which antiepileptics are prescribed are themselves associated with morbidity and mortality and an increased risk of suicidal thoughts and behavior. Should suicidal thoughts and behavior emerge during treatment, the prescriber needs to consider whether the emergence of these symptoms in any given patient may be related to the illness being treated.

Patients, their caregivers, and families should be informed that AEDs increase the risk of suicidal thoughts and behavior and should be advised of the need to be alert for the emergence or worsening of the signs and symptoms of depression, any unusual changes in mood or behavior, or the emergence of suicidal thoughts, behavior, or thoughts about self-harm. Behaviors of concern should be reported immediately to healthcare providers.

\section{Dizziness and Ataxia}

Patients should be advised that VIMPAT may cause dizziness and ataxia. Accordingly, they should be advised not to drive a car or to operate other complex machinery until they are familiar with the effects of VIMPAT on their ability to perform such activities.

In patients with partial-onset seizures taking 1 to 3 concomitant AEDs, dizziness was experienced by $25 \%$ of patients randomized to the recommended doses $(200$ to $400 \mathrm{mg} /$ day) of VIMPAT (compared with $8 \%$ of placebo patients) and was the adverse event most frequently leading to discontinuation $(3 \%)$. Ataxia was experienced by $6 \%$ of patients randomized to the recommended doses (200 to $400 \mathrm{mg} /$ day) of VIMPAT (compared to $2 \%$ of placebo patients). The onset of dizziness and ataxia was most commonly observed during titration. There was a substantial increase in these adverse events at doses higher than $400 \mathrm{mg} /$ day. [see Adverse Reactions/Table 2 (6.1)]

\section{Cardiac Rhythm and Conduction Abnormalities}

\section{PR interval prolongation}

Dose-dependent prolongations in PR interval with VIMPAT have been observed in clinical studies in patients and in healthy volunteers [see Clinical Pharmacology (12.2) in Full Prescribing Information]. In clinical trials in patients with partial-onset epilepsy, asymptomatic first-degree atrioventricular (AV) block was observed as an adverse reaction in $0.4 \%$ (4/944) of patients randomized to receive VIMPAT and $0 \%(0 / 364)$ of patients randomized to receive placebo. In clinical trials in patients with diabetic neuropathy, asymptomatic first-degree AV block was observed as an adverse reaction in $0.5 \%(5 / 1023)$ of patients receiving VIMPAT and $0 \%(0 / 291)$ of patients receiving placebo. When VIMPAT is given with other drugs that prolong the PR interval, further PR prolongation is possible.

VIMPAT should be used with caution in patients with known conduction problems (e.g. marked first-degree AV block, second-degree or higher AV block and sick sinus syndrome without pacemaker), or with severe cardiac disease such as myocardia ischemia or heart failure. In such patients, obtaining an ECG before beginning VIMPAT, and after VIMPAT is titrated to steady-state, is recommended.

\section{Atrial fibrillation and Atrial flutter}

In the short-term investigational trials of VIMPAT in epilepsy patients, there were no cases of atrial fibrillation or flutter in patients with diabetic neuropathy, $0.5 \%$ of patients treated with VIMPAT experienced an adverse reaction of atrial fibrillation or atrial flutter, compared to $0 \%$ of placebo-treated patients. VIMPAT administration may predispose to atrial arrhythmias (atrial fibrillation or flutter), especially in patients with diabetic neuropathy and/or cardiovascular disease. Patients should be made aware of the symptoms of atrial fibrillation and flutter (e.g., palpitations, rapid pulse, shortness of breath) and told to contact their physician should any of these symptoms occur.

\section{Syncope}

In the short-term controlled trials of VIMPAT in epilepsy patients with no significant system illnesses, there was no increase in syncope compared to placebo. In the short-term controlled trials of VIMPAT in patients with diabetic neuropathy, $1.2 \%$ of patients who were treated with VIMPAT reported an adverse reaction of syncope or loss of consciousness, compared to $\% \%$ of placebo-treated patients with diabetic neuropathy. Most of the cases of syncope were observed in patients receiving doses above $400 \mathrm{mg} /$ day. The cause of syncope was not determined in most cases. However, several were associated with either changes in orthostatic blood pressure atrial flutter/fibrillation (and associated tachycardia), or bradycardia.

\section{Withdrawal of Antiepileptic Drugs (AEDs)}

As with all AEDs, VIMPAT should be withdrawn gradually (over a minimum of 1 week) to minimize the potential of increased seizure frequency in patients with seizure disorders.

\section{Multiorgan Hypersensitivity Reactions}

One case of symptomatic hepatitis and nephritis was observed among 4011 subjects exposed to VIMPAT during clinical development. The event occurred in a healthy volunteer, 10 days after stopping VIMPAT treatment. The subject was not taking any concomitant medication and potential known viral etiologies for hepatitis were ruled out. The subject fully recovered within a month, without specific treatment. The case is consistent with a delayed multiorgan hypersensitivity reaction. Additional potential cases included 2 with rash and elevated liver enzymes and 1 with myocarditis and hepatitis of uncertain etiology

Multiorgan hypersensitivity reactions (also known as Drug Reaction with Eosinophilia and Systemic Symptoms, or DRESS) have been reported with other anticonvulsants and typically, although not exclusively, present with fever and rash associated with other organ system involvement, that may or may not include eosinophilia, hepatitis, nephritis, lymphadenopathy, and/or myocarditis. Because this disorder is variable in its expression, other organ system signs and symptoms not noted here may occur. If this reaction is suspected, VIMPAT should be discontinued and alternative treatment started.

\section{ADVERSE REACTIONS}

Because clinical trials are conducted under widely varying conditions, adverse reaction rates observed in the clinical trials of a drug cannot be directly compared to rates in the clinical trials of another drug and may not reflect the rates observed in practice. 
In all controlled and uncontrolled trials in patients with partial-onset seizures, 1327 patients have received VIMPAT of whom 1000 have been treated for longer than 6 months and 852 for longer than 12 months.

\section{Clinical Trials Experience}

\section{Controlled Trials}

\section{Adverse reactions leading to discontinuation}

In controlled clinical trials, the rate of discontinuation as a result of an adverse event was $8 \%$ and $17 \%$ in patients randomized to receive VIMPAT at the recommended doses of 200 and $400 \mathrm{mg} /$ day, respectively, $29 \%$ at $600 \mathrm{mg} /$ day, and $5 \%$ in patients randomized to receive placebo. The adverse events most commonly $(>1 \%$ in the VIMPAT total group and greater than placebo) leading to discontinuation were dizziness, ataxia, vomiting, diplopia, nausea, vertigo, and vision blurred.

\section{Most common adverse reactions}

Table 2 gives the incidence of treatment-emergent adverse events that occurred in $\geq 2 \%$ of aduit patients with partial-onset seizures in the total VIMPAT group and for which the incidence was greater than placebo. The majority of adverse events in the VIMPAT patients were reported with a maximum intensity of 'mild' or 'moderate'.

Table 2: Treatment-Emergent Adverse Event Incidence in Double-Blind, Placebo-Controlled Partial-Onset Seizure Trials (Events $\geq 2 \%$ of Patients in VIMPAT Total and More Frequent Than in the Placebo Group)

\begin{tabular}{|c|c|c|c|c|c|}
\hline $\begin{array}{l}\text { System Organ Class/ } \\
\text { Preferred Term }\end{array}$ & $\begin{array}{c}\text { Placebo } \\
\mathrm{N}=364 \\
\%\end{array}$ & \begin{tabular}{|c|} 
VIMPAT \\
$200 \mathrm{mg} /$ day \\
$\mathrm{N}=270$ \\
$\%$
\end{tabular} & $\begin{array}{c}\text { VIMPAT } \\
400 \mathrm{mg} / \text { day } \\
\mathrm{N}=471 \\
\%\end{array}$ & $\begin{array}{c}\text { VIMPAT } \\
600 \mathrm{mg} / \text { day } \\
\mathrm{N}=203 \\
\%\end{array}$ & $\begin{array}{c}\text { VIMPAT } \\
\text { TOTAL } \\
\mathrm{N}=944 \\
\%\end{array}$ \\
\hline \multicolumn{6}{|c|}{ Ear and labyrinth disorder } \\
\hline Vertigo & 1 & 5 & 3 & 4 & 4 \\
\hline \multicolumn{6}{|l|}{ Eye disorders } \\
\hline Diplopia & 2 & 6 & 10 & 16 & 11 \\
\hline Vision blurred & 3 & 2 & 9 & 16 & 8 \\
\hline \multicolumn{6}{|c|}{ Gastrointestinal disorders } \\
\hline Nausea & 4 & 7 & 11 & 17 & 11 \\
\hline Vomiting & 3 & 6 & 9 & 16 & 9 \\
\hline Diarrhea & 3 & 3 & 5 & 4 & 4 \\
\hline \multicolumn{6}{|c|}{ General disorders and administration site conditions } \\
\hline Fatigue & 6 & 7 & 7 & 15 & 9 \\
\hline Gait disturbance & $<1$ & $<1$ & 2 & 4 & 2 \\
\hline Asthenia & 1 & 2 & 2 & 4 & 2 \\
\hline \multicolumn{6}{|c|}{ Injury, poisoning and procedural complications } \\
\hline Contusion & 3 & 3 & 4 & 2 & 3 \\
\hline Skin laceration & 2 & 2 & 3 & 3 & 3 \\
\hline \multicolumn{6}{|c|}{ Nervous system disorders } \\
\hline Dizziness & 8 & 16 & 30 & 53 & 31 \\
\hline Headache & 9 & 11 & 14 & 12 & 13 \\
\hline Ataxia & 2 & 4 & 7 & 15 & 8 \\
\hline Somnolence & 5 & 5 & 8 & 8 & 7 \\
\hline Tremor & 4 & 4 & 6 & 12 & 7 \\
\hline Nystagmus & 4 & 2 & 5 & 10 & 5 \\
\hline Balance disorder & 0 & 1 & 5 & 6 & 4 \\
\hline Memory impairment & 2 & 1 & 2 & 6 & 2 \\
\hline \multicolumn{6}{|l|}{ Psychiatric disorders } \\
\hline Depression & 1 & 2 & 2 & 2 & 2 \\
\hline \multicolumn{6}{|c|}{ Skin and subcutaneous disorders } \\
\hline Pruritus & 1 & 3 & 2 & 3 & 2 \\
\hline
\end{tabular}

\section{Laboratory abnormalities}

Abnormalities in liver function tests have been observed in controlled trials with VIMPAT in adult patients with partial-onset seizures who were taking 1 to 3 concomitant anti-epileptic drugs. Elevations of ALT to $\geq 3 \times$ ULN occurred in $0.7 \%$ (7/935) of VIMPAT patients and $0 \%(0 / 356)$ of placebo patients. One case of hepatitis with transaminases $>20 x$ ULN was observed in one healthy subject 10 days after VIMPAT treatment completion, along with nephritis (proteinuria and urine casts). Serologic studies were negative for viral hepatitis. Transaminases returned to normal within one month without specific treatment. At the time of this event bilirubin was normal. The hepatitis/nephritis was interpreted as a delayed hypersensitivity reaction to VIMPAT.

\section{Other Adverse Reactions in Patients with Partial-Onset Seizures}

The following is a list of treatment-emergent adverse events reported by patients treated with VIMPAT in all clinical trials in patients with partial-onset seizures, including controlled trials and long-term open-label extension trials. Events addressed in other tables or sections are not listed here. Events included in this list from the controlled trials occurred more frequently on drug than on placebo and were based on consideration of VIMPAT pharmacology, frequency above that expected in the population, seriousness; and likelihood of a relationship to VIMPAT. Events are further classified within system organ class.

Blood and lymphatic system disorders: neutropenia, anemia

Cardiac disorders: palpitations

Ear and labyrinth disorders: tinnitus

Gastrointestinal disorders. constipation, dyspepsia, dry mouth, oral hypoaesthesia General disorders and administration site conditions: irritability, pyrexia, feeling drunk Injury, poisoning, and procedural complications: fall

Musculoskeletal and connective tissue disorders: muscle spasms

Nervous system disorders: paresthesia, cognitive disorder, hypoaesthesia, dysarthria, disturbance in attention, cerebellar syndrome

Psychiatric disorders. confusional state, mood altered, depressed mood

\section{Intravenous Adverse Reactions}

Adverse reactions with intravenous administration generally appeared similar to those observed with the oral formulation, although intravenous administration was associated with local adverse events such as injection site pain or discomfort $(2.5 \%)$, irritation $(1 \%)$, and erythema $(0.5 \%)$. One case of profound bradycardia (26 bpm: BP $100 / 60 \mathrm{mmHg}$ ) was observed in a patient during a 15 minute infusion of $150 \mathrm{mg}$ VIMPAT. This patient was on a beta-blocker. Infusion was discontinued and the patient experienced a rapid recovery.

\section{Comparison of Gender and Race}

The overall adverse event rate was similar in male and female patients. Although there were few non-Caucasian patients, no differences in the incidences of adverse events compared to Caucasian patients were observed.

\section{DRUG INTERACTIONS}

Drug-drug interaction studies in healthy subjects showed no pharmacokinetic interactions between VIMPAT and carbamazepine, valproate, digoxin, metformin, omeprazole, or an oral contraceptive containing ethinylestradiol and levonorgestrel. There was no evidence for any relevant drug-drug interaction of VIMPAT with common AEDs in the placebo-controlled clinical trials in patients with partial-onset seizures [see Clinical Pharmacology (12.3) in Full Prescribing Information)]

The lack of pharmacokinetic interaction does not rule out the possibility of pharmacodynamic interactions, particularly among drugs that affect the heart conduction system.

\section{USE IN SPECIFIC POPULATIONS}

\section{Pregnancy}

\section{Pregnancy Category C}

Lacosamide produced developmental toxicity (increased embryofetal and perinatal mortality, growth deficit) in rats following administration during pregnancy. Developmental neurotoxicity was observed in rats following administration during a period of postnatal development corresponding to the third trimester of human pregnancy. These effects were observed at doses associated with clinically relevant plasma exposures.

Lacosamide has been shown in vitro to interfere with the activity of collapsin response mediator protein-2 (CRMP-2), a protein involved in neuronal differentiation and control of axonal outgrowth. Potential adverse effects on CNS development can not be ruled out.

There are no adequate and well-controlled studies in pregnant women. VIMPAT should be used during pregnancy only if the potential benefit justifies the potential risk to the fetus.

Oral administration of lacosamide to pregnant rats $(20,75$, or $200 \mathrm{mg} / \mathrm{kg} /$ day $)$ and rabbits $(6.25,12.5$, or $25 \mathrm{mg} / \mathrm{kg} /$ day $)$ during the period of organogenesis did not produce any teratogenic effects. However, the maximum doses evaluated were limited by maternal toxicity in both species and embryofetal death in rats. These doses were associated with maternal plasma lacosamide exposures [area under the plasma-time concentration curve; $(\mathrm{AUC})] \approx 2$ and 1 times (rat and rabbit, respectively) that in 
humans at the maximum recommended human dose (MRHD) of $400 \mathrm{mg} /$ day. When lacosamide $(25,70$, or $200 \mathrm{mg} / \mathrm{kg} /$ day) was orally administered to rats throughout gestation, parturition, and lactation, increased perinatal mortality and decreased body weights were observed in the offspring at the highest dose. The noeffect dose for pre- and post-natal developmental toxicity in rats $(70 \mathrm{mg} / \mathrm{kg} /$ day) was associated with a maternal plasma lacosamide AUC approximately equal to that in humans at the MRHD.

Oral administration of lacosamide $(30,90$, or $180 \mathrm{mg} / \mathrm{kg} / \mathrm{day})$ to rats during the neonatal and juvenile periods of postnatal development resulted in decreased brain weights and long-term neurobehavioral changes (altered open field performance, deficits in learning and memory). The early postnatal period in rats is generally thought to correspond to late pregnancy in humans in terms of brain development. The no-effect dose for developmental neurotoxicity in rats was associated with a plasma lacosamide AUC approximately 0.5 times that in humans at the MRHD.

\section{Pregnancy Registry}

UCB, Inc. has established the UCB AED Pregnancy Registry to advance scientific knowledge about safety and outcomes in pregnant women being treated with VIMPAT To ensure broad program access and reach, either a healthcare provider or the patient can initiate enrollment in the UCB AED Pregnancy Registry by calling 1-888-5377734 (toll free).

Physicians are also advised to recommend that pregnant patients taking VIMPAT enrol in the North American Antiepileptic Drug Pregnancy Registry. This can be done by calling the toll free number 1-888-233-2334, and must be done by patients themselves. Information on the registry can also be found at the website http://www.aedpregnancyregistry.org/.

\section{Labor and Delivery}

The effects of VIMPAT on labor and delivery in pregnant women are unknown. In a pre- and post-natal study in rats, there was a tendency for prolonged gestation in al lacosamide treated groups at plasma exposures (AUC) at or below the plasma AUC in humans at the maximum recommended human dose of $400 \mathrm{mg} / \mathrm{day}$.

\section{Nursing Mothers}

Studies in lactating rats have shown that lacosamide and/or its metabolites are excreted in milk. It is not known whether VIMPAT is excreted in human milk. Because many drugs are excreted into human milk, a decision should be made whether to discontinue nursing or to discontinue VIMPAT, taking into account the importance of the drug to the mother.

\section{Pediatric Use}

The safety and effectiveness of VIMPAT in pediatric patients $<17$ years have not been established.

Lacosamide has been shown in vitro to interfere with the activity of CRMP-2, a protein involved in neuronal differentiation and control of axonal outgrowth. Potential adverse effects on CNS development can not be ruled out. Administration of lacosamide to rats during the neonatal and juvenile periods of postnatal development resulted in decreased brain weights and long-term neurobehavioral changes (altered open field performance, deficits in learning and memory). The no-effect dose for developmenta neurotoxicity in rats was associated with a plasma lacosamide exposure (AUC) approximately 0.5 times the human plasma AUC at the maximum recommended human dose of $400 \mathrm{mg} /$ day.

\section{Geriatric Use}

There were insufficient numbers of elderly patients enrolled in partial-onset seizure trials $(n=18)$ to adequately assess the effectiveness of VIMPAT in this population.

In healthy subjects, the dose and body weight normalized pharmacokinetic parameters AUC and $\mathrm{C}_{\max }$ were approximately $20 \%$ higher in elderly subjects compared to young subjects. The slightly higher lacosamide plasma concentrations in elderly subjects are possibly caused by differences in total body water (lean body weight) and age-associated decreased renal clearance. No VIMPAT dose adjustment based on age is considered necessary. Caution should be exercised for dose titration in elderly patients.

\section{Patients with Renal Impairment}

A maximum dose of $300 \mathrm{mg} /$ day is recommended for patients with severe renal impairment ( $\mathrm{CL}_{\mathrm{CR}} \leq 30 \mathrm{~mL} / \mathrm{min}$ ) and in patients with endstage renal disease. VIMPAT is effectively removed from plasma by hemodialysis. Following a 4-hour hemodialysis treatment, AUC of VIMPAT is reduced by approximately $50 \%$.
Therefore dosage supplementation of up to $50 \%$ following hemodialysis should be considered. In all renal impaired patients, the dose titration should be performed with caution. [see Dosage and Administration (2.2) and Clinical Pharmacology (12.3) in Full Prescribing Information]

\section{Patients with Hepatic Impairment}

Patients with mild to moderate hepatic impairment should be observed closely during dose titration. A maximum dose of $300 \mathrm{mg} /$ day is recommended for patients with mild to moderate hepatic impairment. The pharmacokinetics of lacosamide has not been evaluated in severe hepatic impairment. VIMPAT use is not recommended in patients with severe hepatic impairment. [see Dosage and Administration (2.3) and Clinical Pharmacology (12.3) in Full Prescribing Information] Patients with co-existing hepatic and renal impairment should be monitored closely during dose titration.

\section{DRUG ABUSE AND DEPENDENCE}

\section{Controlled Substance}

VIMPAT is a Schedule $V$ controlled substance.

\section{Abuse}

In a human abuse potential study, single doses of $200 \mathrm{mg}$ and $800 \mathrm{mg}$ lacosamide produced euphoria-type subjective responses that differentiated statistically from placebo; at $800 \mathrm{mg}$, these euphoria-type responses were statistically indistinguishable from those produced by alprazolam, a Schedule IV drug. The duration of the euphoriatype responses following lacosamide was less than that following alprazolam. A high rate of euphoria was also reported as an adverse event in the human abuse potential study following single doses of $800 \mathrm{mg}$ lacosamide (15\% [5/34]) compared to placebo $(0 \%)$ and in two pharmacokinetic studies following single and multiple doses of 300 $800 \mathrm{mg}$ lacosamide (ranging from $6 \%$ [2/33] to $25 \%$ [3/12]) compared to placebo $(0 \%)$. However, the rate of euphoria reported as an adverse event in the VIMPAT development program at therapeutic doses was less than $1 \%$.

\section{Dependence}

Abrupt termination of lacosamide in clinical trials with diabetic neuropathic pain patients produced no signs or symptoms that are associated with a withdrawal syndrome indicative of physical dependence. However, psychological dependence cannot be excluded due to the ability of lacosamide to produce euphoria-type adverse events in humans.

\section{OVERDOSAGE}

\section{Signs, Symptoms, and Laboratory Findings of Acute Overdose in Humans}

There is limited clinical experience with VIMPAT overdose in humans. The highest reported accidental overdose of VIMPAT during clinical development was $1200 \mathrm{mg} /$ day which was non-fatal. The types of adverse events experienced by patients exposed to supratherapeutic doses during the trials were not clinically different from those of patients administered recommended doses of VIMPAT.

There has been a single case of intentional overdose by a patient who selfadministered 12 grams VIMPAT along with large doses of zonisamide, topiramate, and gabapentin. The patient presented in a coma and was hospitalized. An EEG revealed epileptic waveforms. The patient recovered 2 days later.

\section{Treatment or Management of Overdose}

There is no specific antidote for overdose with VIMPAT. Standard decontamination procedures should be followed. General supportive care of the patient is indicated including monitoring of vital signs and observation of the clinical status of patient. A Certified Poison Control Center should be contacted for up to date information on the management of overdose with VIMPAT.

Standard hemodialysis procedures result in significant clearance of VIMPAT (reduction of systemic exposure by $50 \%$ in 4 hours). Hemodialysis has not been performed in the few known cases of overdose, but may be indicated based on the patient's clinical state or in patients with significant renal impairment.

\section{PATIENT COUNSELING INFORMATION}

See FDA-approved Medication Guide and Patient Counseling Information section in the Full Prescribing Information.

VIMPAT tablets and VIMPAT injection

\section{$\Gamma$ Manufactured for \\ ust UCB, Inc. \\ - Smyrna, GA 30080}

VIMPAT ${ }^{\circledR}$ is a registered trademark under license from Harris FRC Corporation and covered by one or more claims of U.S. Patent 38,551 .

C 2009 UCB, Inc. All rights reserved. Printed in U.S.A. • VE198-0309
1E 01/2009 UCB, Inc.

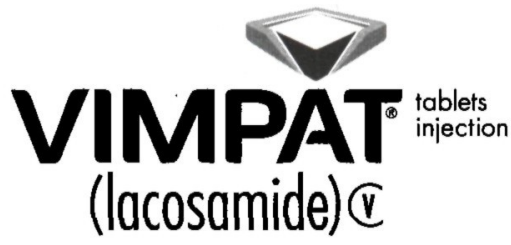

VIMPAT (lacosamide) $\mathbb{C}$ 